\title{
Einbandsammlung Gotthilf Kurz
}

\section{Meisterwerke zeitgenössischer Einbandkunst}

\section{Nachlass Gotthilf Kurz}

Die Württembergische Landesbibliothek Stuttgart verfügt aus dem Nachlass des Buchbinders Gotthilf Kurz (1923-2010) über eine Sammlung von 521 Handeinbänden. Nach Verhandlungen zwischen der Landesbibliothek und den Wissenschaftsministerien in Baden-Württemberg und Bayern kamen die Bände im Juli 2011 in den Stuttgarter Bestand. Sie erweitern erheblich den Fundus an bibliophilen Einbänden zeitgenössischer Künstler. Die meisten davon waren vorher in der Einbandsammlung aus dem Erbe des Stuttgarter Verlegers Max Hettler (1907-1969) zu finden, die im Jahr 2001 in die WLB gelangte. ${ }^{1}$ Als Teil dieser Sammlung befinden sich schon länger fünf von Gotthilf Kurz angefertigte Einbände im Besitz der Landesbibliothek. ${ }^{2}$

Von Interesse für die Württembergische Landesbibliothek ist die Sammlung nicht nur aus bibliophilen Gründen, sondern auch wegen des Regionalbezugs. Der aus Nürtingen stammende Kurz begann 1943 seine Ausbildung an der Werkkunstschule in Offenbach/Main. 1948 absolvierte er die Abschlussprüfung in Schrift und Graphik, 1949 im Buchbinden. Buchgraphische Studien in Stuttgart schlossen sich an. Wichtig für die weitere künstlerische Entwicklung wurde der Aufenthalt am Collège Technique Municipal Estienne in Paris (1955-1956). Seine Prüfungsarbeiten sind in der Sammlung ebenso enthalten wie spätere preisgekrönte Einbände. Nach der Meisterprüfung 1959 war er bis 1965 freiberuflich tätig. Prägend für Generationen angehender Buchbinder wurde er ab 1965 als Leiter der Meisterklasse für Buchbinder an der Akademie für das Graphische Gewerbe München. Von 1967 bis 1982 war Gotthilf Kurz Präsident der 1923 gegründeten Vereinigung Meister der Einbandkunst (MDE). ${ }^{3}$

\section{Stil}

Gotthilf Kurz erweist sich als einer der wichtigsten zeitgenössischen Einbandkünstler durch die große

(1) http://www.wlb-stuttgart.de/sammlungen/alte-und-wertvolle-drucke/ bestand/einbaende/einbandsamm/ung-max-hettler

(2) Signaturen: 51/90051, 51a/90038, 51a/90068, 51/90146, Za 90072 - 3/4.1929/30.

(3) http://www.mde-einbandkunst.eu
Vielfalt der Methoden und Motive sowie die herausragende Qualität seiner Werke. In der Sammlung aus seinem Nachlass sind Einbände mit allen denkbaren Bezugsarten (Leder, Pappe, Gewebe, Pergament, Plexiglas) enthalten.

Am stärksten vertreten sind Leder- (172) und PappEinbände (276). Häufig sind Teilserien mit mehreren nur in der Komposition abweichenden, aber sonst analog strukturierten Einbänden. In einigen Fällen sind drei Bände in einem Schuber zusammengefasst; sie unterscheiden sich z.B. nur durch die geometrische Form des zentralen Dekors (Kreis, Dreieck, Viereck).

Fast alle bekannten Typen von Einbänden sind enthalten. Die Ledereinbände sind überwiegend als Franzband gestaltet. Gerade Altarbibeln und Prachteinbände begegnen häufig in Franzbänden. Die geschlossene, glatte Optik der Franzbände ohne Falzrille sowie die feste Verbindung zwischen Buchblock und Deckel mit der darin begründeten hohen Stabilität des Buches gilt als Qualitätsausweis und unterstreicht die inhaltliche Bedeutung des auf diese Weise gebundenen Buches. Ganzledereinbände verwenden meist Pappe, seltener Holz als Deckelmaterial, häufig Ziegen-, seltener Kalbs- oder Schweinsleder als Bezugsmaterial.

Unter den Pappbänden findet man eine größere Variationsbreite. Pappdeckel mit Lederelementen insbesondere an den Kanten oder auf Teilen des Rückens (Edelpappbände) begegnen ebenso wie orientalische Einbände mit ihrem zweiten, von hinten nach vorne reichenden Vorderdeckel. Ein Interimseinband und viele gewöhnliche Pappbände sind Teil der Sammlung. Kombinierte Materialarten wie Halbleder-, Halbpergament- und Halbgewebebände kommen in kleinerer Zahl vor.

Mehrere Einbände aus dem Nachlass lagen als Prüfungsleistung für die Meisterprüfung oder diverse Wettbewerbe vor. Kurios muten ein rekonstruiertes Beutelbuch, eine bibliophil gestal- 
tete Pergament-Kassette ohne Buchinhalt und die Plexiglas-Einbände mit eingegossenen Elementen wie Goldplättchen oder Holztafeln an.

Die Motivik lässt sich den größeren Gruppen Flächen-, Linien-, Farb- bzw. Schriftdekor zuordnen. Häufig kombiniert Kurz z.B. geometrische Flächen mit gliedernden Linien sowie Farbauftrag. Schriftdekor ist auf dem Rücken häufiger als auf dem Vorderdeckel zu finden. Beispiele für stilisierte inhaltliche Motive (z.B. Landschaft, Bücher) und für symbolische Einbandmotive (z.B. Kreuz, Fische, Chi-Rho) mit Bezug auf den Buchinhalt (sog. redende Einbände) sind vertreten. Zwar nutzt Gotthilf Kurz auch Blindbände, teils in Gestaltung als Gästebücher, für seine buchbinderische Tätigkeit. Häufiger sind jedoch solche inhaltlich definierten Bücher, zu denen potentiell Entsprechungsverhältnisse in der Einbandgestaltung hergestellt werden können. So verwendet er häufig Bibel- oder Teilbibelausgaben (teilweise Pressendrucke), aber auch

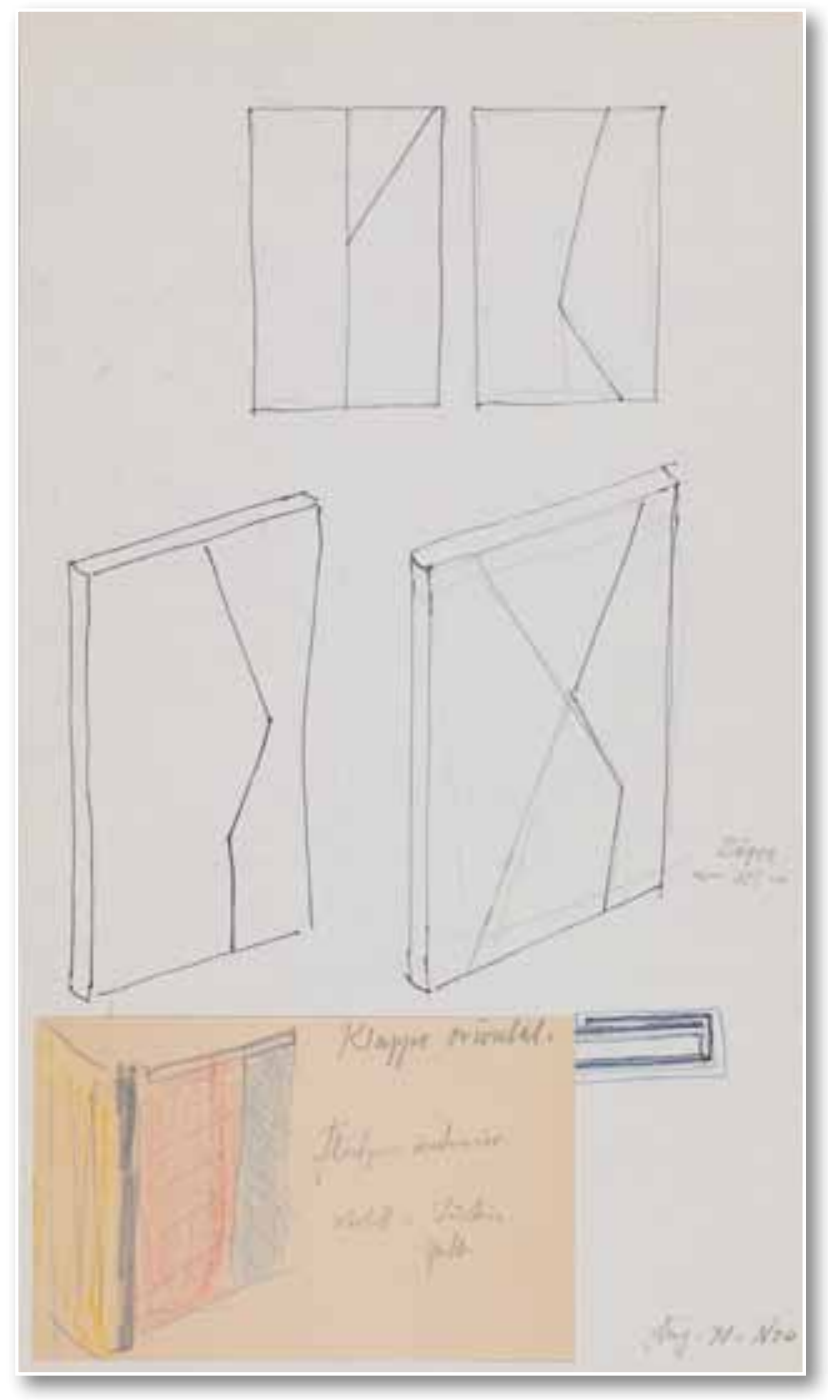

Bild 1: Entwurfszeichnung für einen orientalischen Einband
Klassikertexte (z.B. Homer, Hölderlin) und Werke bedeutender, zumal württembergischer Personen wie Theodor Heuss (1884-1963).

Abstrakt-ornamentale Verzierung liegt Kurz mehr als figürlicher Schmuck. Dementsprechend findet man auf Ledereinbänden insbesondere Blindprägung oder Lederauflagen in unterschiedlichen Farben. Blindprägung dient manchmal auch zur Herstellung von Flächen- oder Liniendekor auf Pappbänden. Wichtiger ist aber der Farbauftrag, der als Aquarell, in Walztechnik, Acrylfarbenauftrag oder im Spachtelverfahren erfolgen kann. Klar voneinander abgetrennte Farbflächen sind seltener als ineinander übergehende bzw. verschwimmende Farben oder die Besetzung einer ganzen Farbpalette (z.B. Erdtöne, Blautöne usw.).

Aufschluss über Arbeitsschritte und Materialien geben auch diverse Entwurfszeichnungen, SchabIonen, Modelle, die von Kurz angefertigt wurden.

\section{Dokumentation}

Für die Dokumentation der einzelnen Einbände sind folgende Kriterien maßgeblich: Übersichtlichkeit, Vollständigkeit, Recherchierbarkeit, Nachhaltigkeit. Abweichend von dem Verfahren bei der Einbandsammlung Hettler und der speziellen Einbandsammlung (ES) wurden daher nicht einzelne PDF- bzw. HTML-Dateien für die jeweiligen Einbände angelegt, die einzeln gepflegt werden müssen. Doppelarbeiten bei der formalen Erfassung der Bände sollten vermieden werden. Vielmehr erfolgt die Erfassung der Einbandsammlung Kurz mit formalen Titeldaten, Provenienzangaben und schematisierter Einbandbeschreibung im SWBKatalog. Die Einbände aus dem Nachlass Kurz werden als geschlossene Nachlass-Sammlung mit einheitlicher Provenienz, eigener Bandzählung und speziellem Abrufzeichen abgebildet. Dies macht ihre Dokumentation unabhängig von der Existenz anderer Exemplare derselben Schriften möglich. Zudem erfolgt die Erfassung exemplarspezifischer Merkmale (Einbandgestaltung) im Bereich der Titeldaten. Dadurch sind die Merkmale der Einbände weltweit über den SWB-Katalog, nicht nur über eine Recherche in den Lokaldaten bzW. - nach einem Import aus dem SWB - über den WLB-OPAC recherchierbar. Die Aspekte der Einbandgestaltung werden in einer an gedruckte Einbandkataloge 
angelehnten, aber in Umfang, Abfolge und Begrifflichkeit schematisierten bzw. normierten Form in der neuen Einbandbeschreibungskategorie 4278 des SWB erfasst. Der Inhalt der Kategorie ist - in absteigender Bedeutung für die Identifikation des Einbandes - unterteilt in die Teilbereiche „Deckel und Rücken" (mit Bezugs- und Deckelart), "Weitere Beschreibung des Einbands" (Kanten, Schnitt, Kapital, Spiegel, Vorsatz), "Sonstiges“ (Eintragungen, Behältnisse, Buchbinder, Land, Entstehungsjahr, Format, Maße, Typ). Sobald diese Daten indexiert wurden, kann mit dem Schlüssel fnm bzw. im Freitext gezielt nach Einzelaspekten übergreifend in der ganzen Sammlung gesucht werden - ein erheblicher Mehrwert gegenüber der Dokumentation mit Einzeldateien. Die Erfassung im SWB hat zudem den Vorteil, dass die Daten durch das BSZ gepflegt und bei Weiterentwicklungen berücksichtigt werden.

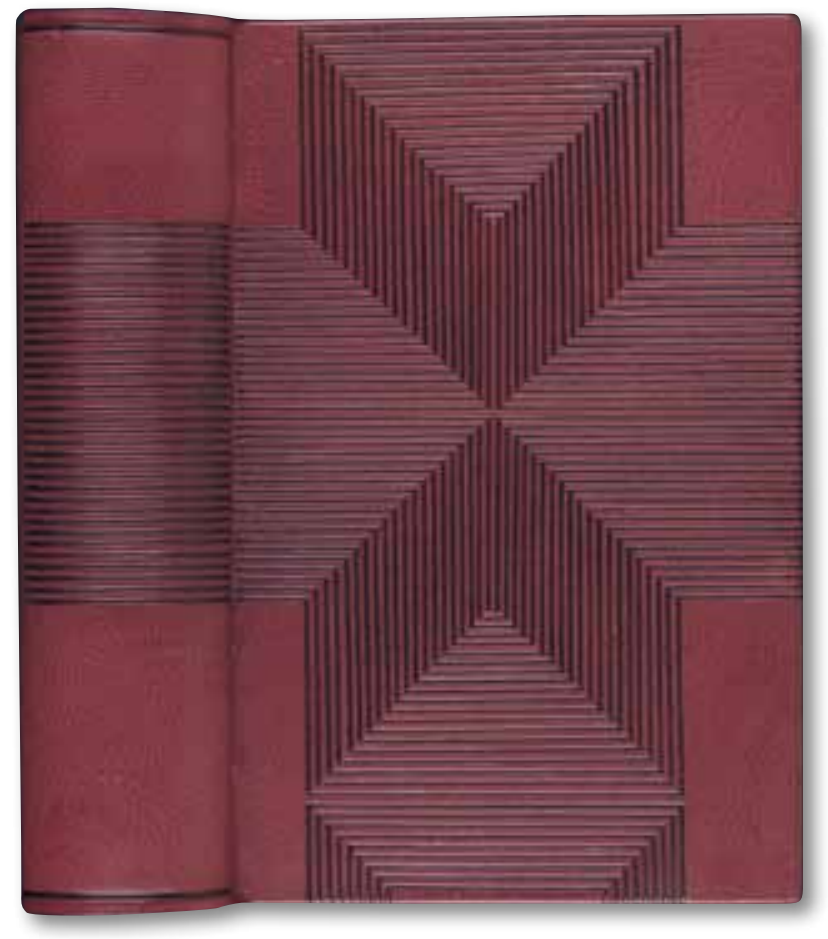

Bild 2 : A35/43: Altarbibel mit rotem Oasenziegenledereinband

Die Sammlung wird komplett, nicht nur exemplarisch oder summarisch beschrieben. Per Link gelangt man zu einem Digitalisat des Vorderdeckels. Von der Großaufnahme des Vorderdeckels aus sowie von der Einführungsseite auf der Homepage ${ }^{4}$ kommt man zu einer Übersicht mit verkleinerten Aufnahmen (Thumbnails) aller Einbände, die per Identnummer mit den jeweiligen SWB-Titelaufnah- men verknüpft sind. ${ }^{5}$ So steht ein eher optischintuitiver Zugang neben einer systematisch-intellektuellen Recherchierbarkeit. Alle Datensätze der Einbandsammlung Kurz verfügen zudem über eine Sacherschließung nach RSWK.

Beispiel - Einbandkategorie (4278) im Datensatz:

4278 Einband\$bBezugsart: Leder (Ziege, Oasenziege) rot (weinrot)\$bDeckelart: Holz\$bBeschreibung Deckel: Ganzledereinband\$bVorderdeckel: Blindprägung, Liniendekor: stilisiertes Kreuz\$bHinterdeckel: wie Vorderdeckel\$bRücken: Blindprägung, Liniendekor\$cSchnitt: berauft\$cKapital: Lederkapital braun (hellbraun): Blindprägung, Liniendekor; Häubchen: Blindprägung, Liniendekor\$cSpiegel: eingelassen; Büttenpapier grau; Ledereinschlag\$cVorsatz: Büttenpapier grau\$dEintragungen: handschriftliche Vermerke, Bleistift: G.Kurz rel. et dor. 1962: fliegender hinterer Vorsatz\$dSchuber: ledergefasst; Büttenpapier (Romabütten) grau (hellgrau) \$dBuchbinder: Gotthilf Kurz\$dLand: Deutschland\$dEntstehungsjahr: 1962\$dFormat: Folio\$dMaße: 34,5 × 22,5 cm\$dTyp: Franzband\$dTyp: Redender Einband\$dTyp: Altarbibel

Die Dokumentation der Einbandsammlung Gotthilf Kurz beruht auf einer Gemeinschaftsleistung mehrerer Personen aus den Bereichen Alte und Wertvolle Drucke, Medienbearbeitung, IT, Digitalisierungswerkstatt. Die Details der Einbandbeschreibung sind als Projektarbeit im Rahmen eines Langzeitpraktikums der Bachelor-Ausbildung an der HdM entstanden. 